\title{
Referring to multimodal rehabilitation for patients with musculoskeletal disorders - a register study in primary health care
}

Charlotte Post Sennehed ${ }^{1,2,3^{*}}$, Sara Holmberg ${ }^{3,4}$, Kjerstin Stigmar ${ }^{2,5}$, Malin Forsbrand ${ }^{1,2,6}$, Ingemar F. Petersson ${ }^{1,2}$, Anja Nyberg ${ }^{7}$ and Birgitta Grahn ${ }^{1,2,3}$

\begin{abstract}
Background: In 2008, the Swedish government introduced a National Rehabilitation Program, in which the government financially reimburses the county councils for evidence-based multimodal rehabilitation (MMR) interventions. The target group is patients of working age with musculoskeletal disorders (MSD), expected to return to work or remain at work after rehabilitation. Much attention in the evaluations has been on patient outcomes and on processes. We lack knowledge about how factors related to health care providers and community can have an impact on how patients have access to MMR. The aim of this study was therefore to study the impact of health care provider and community related factors on referrals to MMR in patients with MSD applying for health care in primary health care.

Methods: This was a primary health care-based cohort study based on prospectively ascertained register data. All primary health care centres (PHCC) contracted in Region Skåne in 2010-2012, referring to MMR were included ( $n=153$ ). The health care provider factors studied were: community size, PHCC size, public or private PHCC, whether or not the PHCCs provided their own MMR, burden of illness and the community socioeconomic status among the registered population at the PHCCs. The results are presented with descriptive statistics and for the analysis, non-parametric and multiple linear regression analyses were applied.

Results: PHCCs located in larger communities sent more referrals/1000 registered population $(p=0.020)$. Private PHCCS sent more referrals/1000 registered population compared to public units $(p=0.035)$. Factors related to more MMR referrals/1000 registered population in the multiple regression analyses were PHCCs located in medium and large communities and with above average socioeconomic status among the registered population at the PHCCs, private PHCC and PHCCs providing their own MMR. The explanation degree for the final model was $24.5 \%$.

Conclusions: We found that referral rates to MMR were positively associated with PHCCs located in medium and large sized communities with higher socioeconomic status among the registered population, private PHCCs and PHCCs providing their own MMR. Patients with MSD are thus facing significant inequities and were thus not offered the same opportunities for referrals to rehabilitation regardless of which PHCC they visited.
\end{abstract}

Keywords: Multiprofessional rehabilitation, Musculoskeletal disorders, Primary health care, Referral rate

\footnotetext{
* Correspondence: charlotte.sennehed@kronoberg.se

${ }^{1}$ Faculty of Medicine, Department of Clinical Sciences Lund, Orthopedics, Lund University, Lund, Sweden

Epidemiology and Register Centre South, Region Skåne, Lund, Sweden

Full list of author information is available at the end of the article
} 


\section{Background}

Musculoskeletal disorders (MSD) are among the most common reasons for sick leave in western countries [1-3]. The Global Burden of Disease Study reported in 2012, that MSD caused $21 \%$ of all years lived with disability and within MSD, the largest group was those with low back pain [4]. In Sweden MSD encompasses the second largest proportion of sick leave. In 2012, 25\% of those on sick leave were so due to these disorders (personal communication, The Swedish Social Insurance Agency) and incidence and costs are increasing [5]. For reincentivizing, i.e. stay at work and return to work, the patient needs strategies to regain physical, mental and social functions [6]. Most patients in Sweden with MSD get their first treatment in primary health care (PHC) and when patients need they can receive further rehabilitation by referral from the primary health care centres (PHCC) to secondary care. In 2012, about $20-30 \%$ of the total numbers of visits in PHC were made by patients with MSD [7] and patients diagnosed with back pain consumed twice as much health care resource as the general population [8].

In 2008, the Swedish government introduced a warranty National Rehabilitation Program with the ambition to provide all inhabitants in Sweden with evidence-based rehabilitation in PHC [9]. Different specialized rehabilitation programmes for long-lasting pain have been evaluated and there is some evidence that multimodal rehabilitation (MMR) is effective in relation to return to work $[10,11]$ and also cost effectiveness has been proven $[12,13]$. When rehabilitation has been combined with work place interventions MMR has been found effective $[14,15]$.

The state financially reimburses the PHC in the county councils for this evidence-based rehabilitation directed to patients in working age, 16-67 years of age, with mild to moderate mental disorders and patients with MSD, mainly neck, shoulder and back pain [16]. The National Rehabilitation program is proposed to strengthen the opportunities for rehabilitation for the two large patient groups at risk for developing long-lasting problems and sick leave [17] and is intended to improve function, work ability and to reduce social costs due to ill health and sick leave.

In Sweden, patients with MSD are offered MMR after referrals in PHC. The rehabilitation can be provided by private or public contracted units all funded by the county councils. To get a referral to MMR the patient has to visit a physician in PHC for medical assessment. Thus it is not possible for the patient to access MMR without referral. MMR involves a multiprofessional team with physician, physiotherapist, psychologist and occupational therapist. MMR is given full or part-time over four to eight weeks and includes cognitive behavioural therapy, physical therapy and patient education. The rehabilitation is mainly provided as group treatments. Therefore it is not possible to get only individual treatment within the MMR programme. For each completed MMR the unit executing the care receives financial compensation. During the first years of the National Rehabilitation Program the county councils was compensated with 45000 SEK (5473 USD) per patient.

The MMR within the National Rehabilitation Program implemented in Swedish PHC has been evaluated with special attention on process, implementation and development. The implementation process has been slow, partly due to ambivalence regarding development of new treatment modalities, organisational uncertainties and ambiguities concerning patient selection [18]. However, the evaluation found that PHCCs experienced opportunities for expanded operations and were positive towards a focus on psychosocial interventions for patients $[19,20]$. Difficulties in implementation were seen, e.g. that MMR teams had inadequate competence concerning rehabilitation focusing work ability and sick-listed patients return to work [18]. In summary, there is lack of evidence regarding early rehabilitation in $\mathrm{PHC}$ for patients with MSD [21].

Much attention in the evaluations of the National Rehabilitation Program has been on patient outcomes [22] and on process $[10,20]$. We know from other contexts, for example regarding use of diagnostic methods and antibiotic treatment, that there are big inexplicable differences between caregivers and geographical areas $[23,24]$. In order to prevent inequity there is a need to know more about health care provider factors (organisational and economic) in relation to referrals and health outcomes. To our knowledge, this is the first time health care provider factors are studied in relation to referrals to MMR. The aim of this study was therefore to study the impact of health care provider and community related factors on referrals to MMR in patients with MSD applying for health care in PHC.

\section{Methods \\ Design}

This was a PHC-based cohort study based on prospectively ascertained register data from the Healthcare Governance in Region Skåne in south Sweden.

\section{Setting}

Almost all health care in Sweden is tax-financed. Both public and private health care providers are available. $\mathrm{PHC}$ is mostly the first care giver instance in Sweden, responsible for all basic healthcare and referrals to appropriate specialist healthcare. Region Skåne has 13\% of Sweden's population and increased from 1243329 inhabitants in year 2010 to 1263088 inhabitants in year 2012. Since 2010, all inhabitants in Region Skåne have 
opportunities to actively choose any public or private accredited PHCC for registration. Patients who do not actively choose a PHCC are registered based on the latest visit or the shortest distance to the PHCC. The PHCCs need to be accredited by the local county council and the reimbursement paid to the PHCC follows the patient. The PHCCs are obligated to participate in follow-ups on quality of care. Measurements of burden of illness and socioeconomic status of the registered population determine the financial compensation for the PHCCs. In Region Skåne, as well in Sweden in general, PHCCs are multiprofessional with physician, nurse, physiotherapist, occupational therapist and psychologist [25].

\section{Data collection}

We obtained permission to use available data from the Healthcare Governance in Region Skåne. No questionnaires were sent to PHC or PHCCs in order to obtain their opinions. Inclusion criteria were all accredited PHCCs contracted in Region Skåne, who had issued any referrals during 2010-2012. We identified 233 health care units in the years 2010 to 2012 that had issued referrals to MMR in PHC.

Number of inhabitants in the community (community size) where the PHCC was located was retrieved from Statistics Sweden. From the Healthcare Governance in Region Skåne we assessed data about number of referrals/PHCC, registered population/unit (PHCC size), model of health care (public or private PHCC), whether or not the PHCC provided their own MMR team (internal/external MMR) burden of illness/burden of morbidity (ACG) and socioeconomic status (CNI).

Data from the Regional Council Skåne and Statistics Sweden were input manually into a SPSS 20.0 database for analysis. Data quality was thoroughly checked and validated.

Excluded units were clinics in specialist care such as psychiatry, occupational health and individual health care providers not incorporated in the PHC organisation and hence not accredited. The majority of these excluded units had only occasional referrals to MMR over the three years and the referrals were returned to remittance. Another two units were excluded due to missing data and starting up the unit in late 2012, just a few days before the inclusion was closed. The final analysis therefore included 153 PHCCs (Fig. 1).

\section{Outcome}

The outcome was number of referrals to MMR/1000 registered in the population at the PHCC (referral rate).

\section{Independent variables}

Independent variables operationalising health care provider factors and community were chosen based on

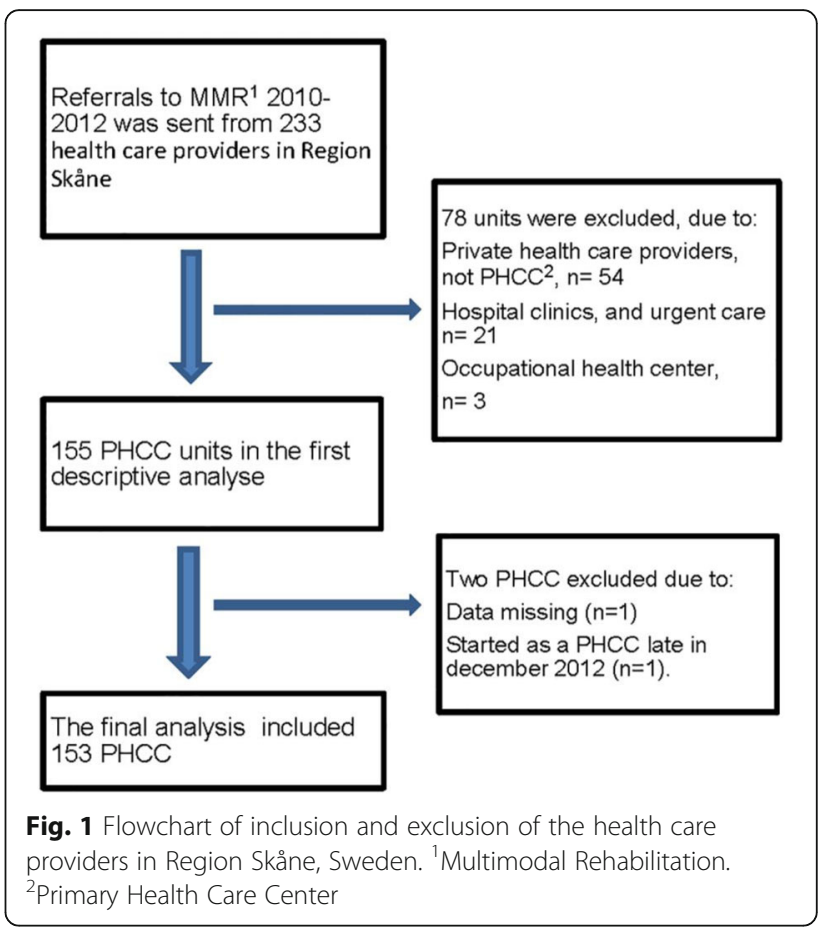

clinical and organisational experience, in conjunction with previous research findings and limitations.

The accessibility of health care services varies with geographical location, due to distances but also due to fewer options to choose care providers in less populated areas [26-28]. In Sweden fewer new PHCCs are established in areas with lower socio-economic status [26]. Geographical related access to PHC might therefore impact referral to MMR [26]. Studies have shown that PHCCs size may affect access to health care and how patients experience the quality of healthcare regarding accessibility of care, consultation length and continuity $[29,30]$. Smaller PHCCs have better accessibility and better continuity of care compared with larger units. In a Swedish report the proportion of various referrals per PHCC varied with unit size [30]. New PHCCs in Sweden are almost exclusively privately operated and are established primarily in urban areas [26]. The introduction of freedom of choice in PHC has shifted care to become more customer-oriented [31]. Since patients attending private units generally have better health, $[32,33]$ there is a risk of inequity. Therefore the organisational model of health care such as the PHCCs providing their own MMR might impact referrals. The reimbursement to the PHCCs follows the patient and the PHCCs need to finance their operations [26]. To provide in-depth rehabilitation, such as MMR, can provide great additional income for the PHCC $[19,20]$. This might be a financial incentive for more referrals.

Burden of illness/burden of morbidity is based on Adjusted Clinical Groups (ACG) and was developed by 
the School of Hygiene and Public Health at John Hopkins University in Baltimore, USA [34, 35]. ACG can be a useful instrument to describe the disease patterns, age and gender in a population [36]. For each PHCC a weighted ACG value is calculated based on recorded diagnoses among its patients and used to estimate the financial compensation to each PHCC. The mean value is 1.0. Values above 1.0 indicate increased morbidity and health care burden. This is important as the patients' type of illness, degree of illness and morbidity at the PHCC might affect how referrals are written.

Socioeconomic status among the registered population at the PHCCs is based on Care Need Index (CNI), which assess health risk indicators for populations [37]. CNI includes the following criteria: elderly persons living alone, children under age 5 , unemployed, low education, single parents, recently moved and born in another country. For each PHCC a weighted CNI is calculated based on its registered population and is used to additionally calculate the financial compensation to each PHCC within the region [37, 38]. Values above 1.0 indicate a lower socioeconomic status and higher risk of developing illness in the defined population. Populations with higher socioeconomic status generally have better health and are more likely to request care [32, 33]. This might impact health professionals' tendency to provide referrals.

\section{Statistical analysis}

Descriptive statistical analyses for the three years were made by percentage, median and quartiles. In order to do statistical analysis, the independent variables were grouped according to criteria described below. The cut off values were decided using a pragmatic approach based on the a priori hypotheses in combination with the data generated. Concerning community size of PHCC location the variables was divided in relatively equal groups relevant to Swedish demography, very small $(<10000$ inhabitants), small (10 000-34 999 inhabitants), medium (35 000-100 000 inhabitants) and large (>100 000 inhabitants) in the community. The variables concerning PHCC size were grouped as small ( $<6000$ registered population), medium (6000-10 500 registered population) and large (>10 500 registered population). This division matches with Swedish standard and was relevant in the analysis. There were large variance regarding the PHCCs ACG (0.69-1.37) and CNI (0.53-2.44) values in line with previous studies $[23,30]$. For the nonparametric tests and the multiple linear regressions analyses we needed to do sub-grouping, in order to do comparisons between higher and lower values. The value regarding burden of illness/ACG varied around 1.0. We categorized the units in three groups, PHCCs with lower $(<0.95)$, medium $(0.95-1.05)$ and higher $(>1.05)$ burden of illness based on the distribution of ACG among the PHCCs. Socioeconomic status/CNI also varied around 1.0. We categorized the units in three groups, higher $(<0.95)$, medium $(0.95-1.05)$ and lower socioeconomic status (>1.05) based on the distribution of CNI among the PHCCs. Values below and above the medium groups indicate higher or lower burden of illness and higher or lower socioeconomic status, and therefore above or below normal. We used nonparametric tests to analyze the distribution of referral rate for each variable; PHCC location, PHCC size, whether or not the PHCC provided their own MMR, ACG and CNI. The Kruskal-Wallis test was used to analyze group differences for PHCCs location and size, ACG and CNI. The Mann-Whitney test was used when comparing public and private PHCCs and whether or not the PHCC provided their own MMR. Finally, multiple linear regression analyses were performed to find factors independently associated with referral rate/1000 registered population to MMR. In the nonparametric tests and the multiple linear regression analyses we used data from 2012. The reason for this was that MMR in Region Skåne was introduced in late 2009 and our intention was to study referrals to MMR after implementation had been stabilized. To achieve the final model, significant variables were provided from a stepwise procedure with $p<0.05$ as inclusion criterion and $p>0.1$ as the removal (of already included variables) criterion. Variables excluded by the stepwise regression model were PHCC size, ACG and the interaction term private PHCC and internal MMR. There were no other interaction terms identified. Since the independent variables were categorical unstandardized B were provided, as they directly indicate/represent the difference of referring/1000 inhabitants between one category/group and another. All variables were analyzed and finally community size where the PHCCs were located and socioeconomic status among the registered population at the PHCCs, private PHCCs and internal MMR at the PHCCs were put in the regression model. Constant was very small and small communities, small communities with lower socioeconomic status, public PHCCs and PHCCs with external MMR. $P$-values less than 0.05 were considered significant.

\section{Ethical considerations}

This study has been approved by the Regional Ethical Review Board in Lund, Dnr 2014/290. In Region Skåne the PHCCs are obligated to participate in follow-ups on quality of care.We had agreements from the Regional Council Skåne to evaluate and study health care provider factors associated to referring to MMR.

\section{Results}

PHCCs size varied with a registered population that ranged from 678 to 24254 . The care burden measured by ACG and CNI varied significantly between PHCCs 
(Table 1). A larger number of PHCCs were situated in very small communities with less than 10000 inhabitants $(38 \%)$ compared to large communities with more than 100000 inhabitants (17\%). Of the 153 PHCCs included, 89 (58\%) were public units and 64 (42\%) were private units. Thirty-seven (24\%) PHCCs had internal MMR teams. Sixty-six PHCCs (45\%) had a medium burden of illness (ACG value 0.95-1.05) and 89 PHCCs (60\%) had higher socioeconomic status with low CNI value $(<0.95)$ (Table 2). In 2010, five PHCCs did not send any referral to MMR. In 2011, two PHCCs did not send any referral to MMR and in 2012 four PHCCs did not send any referral to MMR. During the whole data collection period, two PHCCs were identified that had not sent any referrals to MMR. We excluded 78 units not defined as PHCCs; they were not incorporated in the $\mathrm{PHC}$ organisation and were not accredited.

The total number of sent MMR referrals from all PHCCs $(n=153)$ included in the analysis increased from 6078 referrals in 2010 to 13153 sent referrals in 2012 (Table 3). Referral rate/1000 registered population increased from median 3.2 in 2010 to median 7.8 in 2012 (Table 3) and (Fig. 2a-c).

Nonparametric tests showed that PHCCs situated in larger communities sent more referrals/1000 registered population than those in smaller communities $(p=0.020)$ Private PHCCs sent more referrals/1000 registered population compared to public PHCCs, median 12.6 vs. 7.2 $(p=0.035)$ (Table 4).

The multiple linear regression analyses revealed that the referral rate was higher among PHCCs located in medium and large communities with higher socioeconomic status among the registered population. Referral rate was higher among private PHCCs and PHCCs with internal MMR regardless of community size and socioeconomic status (Table 5). The coefficient of determination for the final model was $\mathrm{R}^{2}=24.5 \%$.

\section{Discussion}

Patients with MSD are facing significant inequities and were thus not offered the same opportunities for referrals to MMR regardless of which PHCC they visited. Our analysis showed that health care provider related factors were significantly associated with referral rate to MMR in PHC. Sent referrals/1000 registered population to MMR was positively associated with PHCCs located in medium and large communities with higher socioeconomic status among the registered population, private PHCCs and PHCCs with internal MMR.

Previous research on MMR has focused on patient related factors, but one can expect that outcomes may also depend on factors related to the health care system. According to our results health care providing factors such as PHCC location, PHCC size, public or private PHCC, whether or not the PHCCs provided their own MMR treatment, the burden of illness and the socioeconomic status in the area are of importance for access to rehabilitation. Differences in attitudes to MMR might also be important, but this could not be captured by the present study design, since this study is based on register data only. The results shed light on factors related to referral rates to MMR and indicate that patients with MSD were not offered the same opportunities for rehabilitation depending on which PHCC they visited. If there are differences in who actually is offered MMR, this might have an impact also on patient outcomes after MMR.

The analysis revealed differences between public and private PHCCs in the referral rate to MMR. We believe that this could be related to private PHCCs having more experience and knowledge of MMR and which patients that would benefit from the treatment modality. Patients in private PHCCs might more frequently request MMR, or can be more motivated and/or have the resources to participate in MMR. Participation in the MMR requires motivation, the opportunity to be on sick leave and the

Table 1 Characteristics of $\mathrm{PHCCs}^{\mathrm{a}}$ in Region Skåne that had prescribed referrals to MMR ${ }^{\mathrm{b}}$ in 2010-2012

\begin{tabular}{|c|c|c|c|c|c|c|c|c|c|c|c|c|}
\hline & \multicolumn{4}{|c|}{2010} & \multicolumn{4}{|c|}{2011} & \multicolumn{4}{|c|}{2012} \\
\hline & $n^{f}$ & Md & $\mathrm{Q1} / \mathrm{Q3}^{\mathrm{e}}$ & Min/Max & $n^{f}$ & Md & Q1/Q3 & Min/Max & $\mathrm{n}^{f}$ & $\mathrm{Md}$ & $\mathrm{Q}_{1 / \mathrm{Q}}{ }^{\mathrm{e}}$ & Min/Max \\
\hline $\begin{array}{l}\text { PHCCs size, registered } \\
\text { population, total }\end{array}$ & 126 & 7896 & $5456 / 11306$ & $790 / 21456$ & 148 & 7993 & $5418 / 11118$ & $724 / 24254$ & 148 & 8101 & 5690/10 962 & $678 / 21861$ \\
\hline $\begin{array}{l}\text { Burden of illness, } \text { ACG }^{c} \\
\text { Mean }=1.0>1.0 \text { increased } \\
\text { burden of illness }\end{array}$ & 126 & 1.00 & $0.93 / 1.06$ & $0.69-1.49$ & 148 & 1.00 & 0.93/1.07 & $0.68-1.41$ & 148 & 0.99 & $0.94 / 1.06$ & $0.69-1.37$ \\
\hline $\begin{array}{l}\text { Socioeconomic status, } \mathrm{CNI}^{\mathrm{d}} \text {, } \\
\text { Mean }=1.0,>1.0 \text { worse } \\
\text { socioeconomic status }\end{array}$ & 126 & 1.03 & $0.90 / 1.20$ & $0.57-2.01$ & 148 & 1.00 & $0.89 / 1.17$ & $0.59-2.51$ & 148 & 0.91 & $0.83 / 1.07$ & $0.53-2.44$ \\
\hline $\begin{array}{l}\text { a Primary health care center } \\
\mathrm{b}^{\mathrm{b}} \text { Multimodal rehabilitation } \\
{ }^{\mathrm{c}} \text { Adjusted Clinical Groups } \\
{ }^{\mathrm{d}} \text { Care Need Index } \\
{ }^{\text {e }} \text { Range for quartile } 1 \text { and quar } \\
{ }^{\mathrm{f}} \text { Missing PHCCs in year } 2010=\end{array}$ & 3 & 2011 & year $2012=$ & & & & & & & & & \\
\hline
\end{tabular}


Table 2 Characteristics of $\mathrm{PHCCs}^{\mathrm{a}}$ in Region Skåne that had prescribed referrals to $\mathrm{MMR}^{\mathrm{b}}$ in 2012

\begin{tabular}{llll}
\hline & $n^{\mathrm{e}}$ & $\%$ & Missing \\
\hline PHCC location, size grouped & 58 & 38 \\
Very small community < 10000 inhabitants & 33 & 22 \\
Small community 10 000-34 999 inhabitants & 36 & 23 \\
Medium community 35 000-100 000 inhabitants & 26 & 17 \\
Large community > 100 000 inhabitants & & & \\
PHCC size, registered population & 44 & 29 & 5 \\
Small (<6 000) & 57 & 37 \\
Medium (6 000-10 500) & 47 & 31 \\
Large (> 10 500) & & & \\
Model of Health Care & 89 & 58 \\
Public PHCCs & 64 & 42 \\
Private PHCCs & & & \\
PHCCs providing their own MMR & 37 & 24 \\
Yes & 116 & 76
\end{tabular}

aPrimary Health Care Centre

${ }^{\mathrm{b}}$ Multimodal rehabilitation

'Adjusted Clinical Groups

${ }^{\mathrm{d}}$ Care Need Index

eNumber of PHCCs

ability to participate in theoretical parts. About 1100 PHCCs are accredited in Sweden and about 33\% are private. There is a rapid change in Sweden with an increased number of private PHCCs, although tax financed. [25]. The reimbursement paid to PHCCs follows the patient and can be a factor of importance. A large number of registered patients and high flow of assessments might be an incentive to improve the economy of the PHCC. We have not found studies analyzing differences between private and public PHCCs and this is a future research of importance. Private PHCCs were in this study in many cases more recently established compared to public PHCCs and the registered population in private PHCCs had higher socioeconomic status compared to the registered population at public PHCCs [39]. Patients with higher socioeconomic status may have better prerequisites to choose among health care
Table 3 Number of referrals to MMR ${ }^{a}$ from included $\mathrm{PHCCs}^{\mathrm{b}}$ in year 2010-2012 in Region Skåne

\begin{tabular}{|c|c|c|c|c|}
\hline & $n^{c}$ & $M d^{d}$ & Q1/Q3 & Min/Max \\
\hline \multicolumn{5}{|l|}{ Referrals to MMR, total } \\
\hline 2010,148 PHCC, missing $=5$ & 6078 & 24 & $9 / 61$ & $0-327$ \\
\hline 2011,151 PHCC, missing $=2$ & 9998 & 40 & 23/89 & $0-363$ \\
\hline 2012,149 PHCC, missing $=4$ & 13153 & 65 & $29 / 117$ & $1-383$ \\
\hline \multicolumn{5}{|c|}{ Referrals to MMR/1000 registered population } \\
\hline 2010,125 PHCC, missing $=28$ & 675 & 3 & $2 / 7$ & $0-31$ \\
\hline $2011,147 \mathrm{PHCC}$, missing $=6$ & 1150 & 6 & $3 / 11$ & $0-30$ \\
\hline 2012,148 PHCC, missing $=5$ & 1538 & 8 & $4 / 15$ & $0-66$ \\
\hline
\end{tabular}

aultimodal rehabilitation

bPrimary Health Care Center

'Referrals from included PHCCs

${ }^{\mathrm{d}}$ Median, referrals from included PHCCs

${ }^{\mathrm{e}}$ Range for quartile 1 and quartile 3

alternatives and also being able to choose among different rehabilitation alternatives. This is confirmed in an earlier report which focuses on Swedish patients' ability to register which PHCC they would like to be part of [40]. Women, highly educated and young people more often changed or considered to change PHCCs, while the less-educated and people living in smaller communities were less inclined to change PHCC [40]. Furthermore an unjustified difference between population groups often arises in the meeting between patients and health care professionals and therefore medical professionals have an important role of being aware of this and working towards a more equal health care [41].

Residential areas with lower socioeconomic status, in which patients may not visit the PHCC because of financial difficulties, might lead to differences in health care despite major illnesses in the patient groups. Another reason for differences in health care could be patients' ability to influence the care given, propensity to make demands and ability to understand and process the information given [38, 42]. Large financial compensation for completed MMR has probably been a motivating factor for referring to MMR and this might also account for the higher referral rates among units with internal MMR. However, our results should be viewed with caution as the introduction of ACG and CNI to suit Swedish conditions has required a lot of redevelopment [43]. We had no access to diagnosis data and could not analyse number of referrals in relation to number of diagnosed registered population. Therefore the outcome referrals/1000 registered was chosen.

The study has been designed and undertaken, based on high quality data. A strong advantage is that the data covers an entire county council area. After meticulous checking of data we believe that all potential accredited PHCC in the area were included. Only two PHCCs not sending any referrals at all to MRR over the period were 


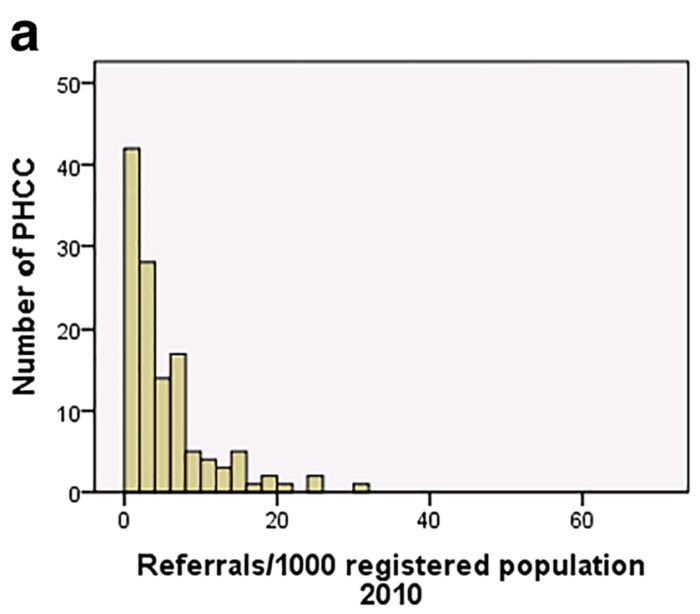

PHCC, $\mathrm{n}=125$

Median $=3.2$

$\mathrm{Q} 1 / \mathrm{Q} 3=1.7 / 7.3$

b

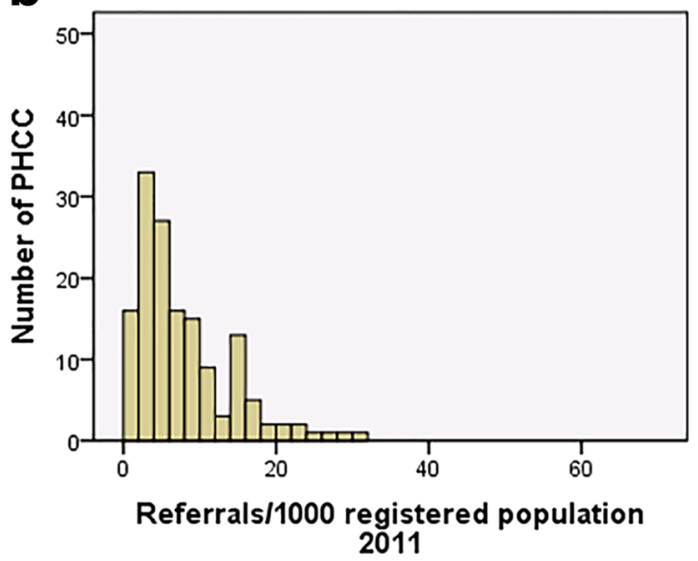

PHCC, $n=147$

Median $=5.9$

$\mathrm{Q} 1 / \mathrm{Q} 3=3.1 / 10.9$

C

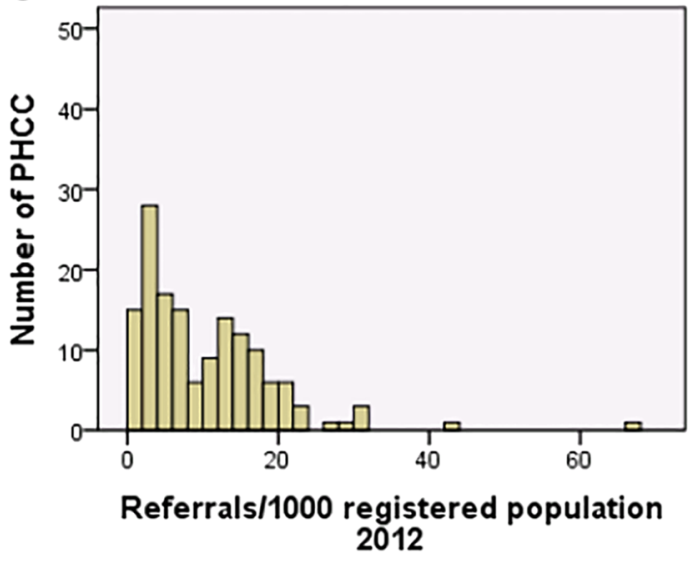

PHCC, $\mathrm{n}=148$

Median $=7.8$

$\mathrm{Q} 1 / \mathrm{Q} 3=3.7 / 15.4$

Fig. 2 a Number of sent referrals/1000 registered population at the PHCCs in 2010. b Number of sent referrals/1000 registered population at the PHCCS in 2011. c Number of sent referrals/1000 registered population at the PHCCs in 2012

identified. Region Skåne has 13\% of Sweden's population and is in many ways representative for Sweden in terms of socio-demographic variables. As far as we know this is the first study about health care provider factors related to referral rate to MMR and we have not found any comparable analysis within PHC. We estimate that the study size is large enough to provide valid results. We found that PHCCs in the largest community and private PHCCs send more referrals/1000 registered population. This is in opposition to the Swedish model where everyone should be offered equivalent care [9]. In the future, health care providers need to be aware that 
Table 4 Number of referrals to $\mathrm{MMR}^{\mathrm{a}} / 1000$ registered population at the PHCCs ${ }^{\mathrm{b}}$ according to health care provider factors in 2012

\begin{tabular}{|c|c|c|c|}
\hline & $M d^{e}$ & Q1/Q3 ${ }^{f}$ & $p$ \\
\hline PHCC location, community size, grouped & & & $0.020^{9}$ \\
\hline $\begin{array}{l}\text { Referrals from very small community }<10000 \text { inhabitants, PHCCs } n=56 \\
\text { Referrals from small community } 10000-34999 \text { inhabitants, PHCCs } n=33 \\
\text { Referrals from medium community } 35000-100000 \text { inhabitants, PHCCs } n=35 \\
\text { Referrals from large community }>100000 \text { inhabitants, PHCCs } n=24\end{array}$ & $\begin{array}{l}6.1 \\
7.8 \\
10.0 \\
16.9\end{array}$ & $\begin{array}{l}6.1 / 12.2 \\
3.2 / 15.0 \\
4.6 / 16.4 \\
4.6 / 20.8\end{array}$ & \\
\hline PHCC size & & & $0.614^{9}$ \\
\hline $\begin{array}{l}\text { Referrals from small PHCCs ( }<6000 \text { registered population), PHCCs } n=44 \\
\text { Referrals from medium PHCCs (6000-10 } 500 \text { registered population), PHCCs } n=57 \\
\text { Referrals from large PHCCs ( }>10500 \text { registered population), PHCCs } n=47\end{array}$ & $\begin{array}{l}6.5 \\
8.1 \\
9.2\end{array}$ & $\begin{array}{l}3.3 / 15.4 \\
4.4 / 14.4 \\
3.5 / 15.8\end{array}$ & \\
\hline Model of health care, public or private PHCC & & & $0.035^{\mathrm{h}}$ \\
\hline $\begin{array}{l}\text { Referrals from public PHCCs, PHCCs } n=86 \\
\text { Referrals from private PHCCs, PHCCs } n=62\end{array}$ & $\begin{array}{l}7.2 \\
12.6\end{array}$ & $\begin{array}{l}3.8 / 13.0 \\
12.6 / 17.6\end{array}$ & \\
\hline Referrals from PHCC providing its own MMR, yes or no & & & $0.111^{\mathrm{h}}$ \\
\hline $\begin{array}{l}\text { Referrals from PHCCs providing their own MMR, no, PHCCs } n=112 \\
\text { Referrals from PHCCs providing their own MMR, yes, PHCCs } n=36\end{array}$ & $\begin{array}{l}7.6 \\
11.8\end{array}$ & $\begin{array}{l}3.5 / 14.0 \\
3.8 / 17.4\end{array}$ & \\
\hline Burden of illness, $\mathrm{ACG}^{\mathrm{C}}$ & & & $0.476^{9}$ \\
\hline \multicolumn{4}{|l|}{ Mean $=1.0>1.0$ increased burden of illness } \\
\hline $\begin{array}{l}\text { Referrals from PHCCs with low ACG }(<0.95) \text {, PHCCs } n=42 \\
\text { Referrals from PHCCs with medium ACG }(0.95-1.05,) \text { PHCCs } n=66 \\
\text { Referrals from PHCCs with high ACG }(>1.05), \text { PHCCs } n=40\end{array}$ & $\begin{array}{l}9.4 \\
6.1 \\
9.7\end{array}$ & $\begin{array}{l}3.6 / 16.8 \\
3.5 / 14.4 \\
3.9 / 14.4\end{array}$ & \\
\hline \multicolumn{4}{|l|}{ Socioeconomic status, $\mathrm{CNI}^{\mathrm{d}}$} \\
\hline Mean $=1.0,>1.0$ worse socioeconomic status & & & $0.322^{9}$ \\
\hline $\begin{array}{l}\text { Referrals from PHCCs in community with low CNI }(<0.95), \text { PHCCs } n=89 \\
\text { Referrals from PHCCs in community with medium CNI }(0.95-1.05), \text { PHCCs } n=18 \\
\text { Referrals from PHCCs in community with high CNI }(>1.05), \text { PHCCs } n=41\end{array}$ & $\begin{array}{l}7.6 \\
13.8 \\
7.2\end{array}$ & $\begin{array}{l}3.8 / 14.8 \\
4.2 / 18.7 \\
3.3 / 15.5\end{array}$ & \\
\hline
\end{tabular}

${ }^{\mathrm{a}}$ Multimodal rehabilitation, ${ }^{\mathrm{b}}$ Primary Health Care Center, ${ }^{\mathrm{C}}$ Adjusted Clinical Groups, ${ }^{\mathrm{d} C a r e}$ Need Index, ${ }^{\mathrm{e}}$ Median, ${ }^{\mathrm{f}}$ Range for quartile 1 and quartile $3,{ }^{\mathrm{g}} \mathrm{Kruskal}-\mathrm{Wallis}$ Test, ${ }^{\text {h} M a n n-W h i t n e y ~ U ~ T e s t ~}$

all patients should have the same opportunities to be offered evidence based pain rehabilitation regardless of where they live, socioeconomic status and what PHCC they choose. The coefficient of determination for the final model was remarkable high $\left(R^{2}=24.5 \%\right)$. This high level is unusual in studies with this type of data, but in this study we found strong correlation between health care provider factors, community factors and referring rate to MMR.

In the nonparametric tests and the multiple linear regression analyses we used data from 2012 only. The reason for this was that $M M R$ in Region Skåne was introduced in late 2009 and our intention was to study referrals to MMR after implementation had been stabilized, which can be seen in Tables 1 and 3. In 2012, the PHCCs had developed procedures and working methods, which also included screening instruments for identification of patients in need of MMR. The number of referrals from units without PHCC contracts decreased over time. In 2010, 52 units with no contracts sent 116 referrals and in 2012, eight units with no contracts sent 37 referrals. Implementation takes time and it is difficult organising new ways of working [18, 20, 44-46].

Table 5 Health care provider factors independently related to referral rate to multimodal rehabilitation

\begin{tabular}{|c|c|c|c|c|}
\hline \multirow[b]{2}{*}{ Model $^{a}$} & \multirow[b]{2}{*}{$B^{C}$} & \multicolumn{3}{|c|}{$95 \%$ confidence interval for B } \\
\hline & & Lower bound & Upper bound & $p$ \\
\hline (Constant) $^{b}$ & 5.36 & 3.26 & 7.46 & $<0.001$ \\
\hline 35-100 000 inhabitants with higher socioeconomic status & 4.88 & 1.15 & 8.61 & 0.011 \\
\hline >100 000 inhabitants with higher socioeconomic status & 16.98 & 10.44 & 23.51 & $<0.001$ \\
\hline >100 000 inhabitants with lower socioeconomic status & 7.29 & 3.26 & 11.31 & $<0.001$ \\
\hline PHCC, private model of health care & 3.95 & 1.33 & 6.57 & 0.003 \\
\hline PHCC, provides its own MMR & 4.41 & 1.35 & 7.47 & 0.005 \\
\hline
\end{tabular}

Multiple regression analysis, $\mathrm{R}^{2}=0.245$

${ }^{b}$ Constant was very small and small communities, communities with 10 000-35 000 inhabitants with lower socioeconomic status, PHCC public model of health care and PHCC not providing its own MMR

'Unstandardized B coefficient 
Difficulties in implementation and measurements are also confirmed in studies about organisation, work practices and evaluations in healthcare quality. Professionals motivation, values, behaviours and interactions with patients is a prerequisite to manage quality in health care [47]. Accounting for quality in healthcare can be problematic and can result in counterproductive effects when it is not fully understood [48]. Phillips et al [49] describes another measurement for health care organisations including interviews and observations to assess the quality in health care. Changes in the methods used and how work is organised in health care could lead to a better use of different competencies. Thereby professionals could focus on the right duties and areas within their competence [50].

The findings in our study can be helpful for different health care organisation, such as county councils, to ensure that health care is delivered and available for all inhabitants; in the end to prevent health inequity. However this study does not provide information on which type of rehabilitation is best for different patients' needs. From a clinical perspective, equal care is not the same as equal rehabilitation for every patient. Professionals need to develop better methods to achieve individual precision in rehabilitations. Screening methods evaluating socioeconomic, psychosocial and physical factors might help health care professionals to individualise the treatment and thereby improving functions and work ability $[51,52]$. A limitation in the study is that we have no knowledge about pain patients not offered MMR and if they were offered other customized pain treatments/ rehabilitations. No comparison has been made between PHCCs, which recently started compared to PHCCs with long experience and established routines in the team.

This study focuses on organisational level and on different PHCCs in a region in Sweden. Important limitations are that we have no data on details regarding the provided MMR. Discussions or recommendations regarding MMR taking place between patient and physician and whether the patient is expected to benefit from MMR was not captured. Furthermore, PHCCs internal working methods, staff conditions and teamwork have not been analysed due to lack of data, factors which may also be of importance. Likewise data concerning competence among personnel were not available, which therefore could not be taken in to account in this study, but might be important in future research.

Other health care provider factors such as physicians employed by staffing companies, temporary staff and staff turnover at the PHCCs have not been analyzed. These types of factors might also influence referral rates to MMR, but we had no such data available. However, a prerequisite for being assigned to deliver MMR care in Region Skåne is that all competence requirements and implementation of MMR are full-filled.
Equal care is a national goal in Swedish healthcare and patients in need should be offered MMR regardless of which PHCC they visit. Future analysis can provide more details on to what extent patients attended MMR at the same unit as made the referral and if this affect treatment outcome. The results in this study is based on a total cohort from Region Skåne, which, concerning sociodemographic factors, very much correspond to Sweden as a whole. This might indicate that these results are possible to generalize.

In this study we had an organisational perspective and the aim was to study health care provider factors impact on referring rate to MMR. We had no possibility to analyse separate socioeconomic status among the registered population such as income, unemployment, sick leave or care consumption in different areas in Region Skåne, since no individual level data were available. The final regression analyse showed the complexity between factors impact on referral to MMR, such as socioeconomic status, PHCC location/ community size, and whether or not the PHCC provided their own MMR. The importance of organisational factors is confirmed in several recent studies [18, 47, 49].

The overall aim of the National Rehabilitation Program is to improve work ability among patients with mild to moderate mental disorders or MSD. Factors found to be important predictors of sick leave and disability pension due to MSD are employment and social relations [53], stress and workload [54, 55], lifestyle and work factors [56], emotional stability and personality [57]. Future studies will investigate interactions between patient related factors and organisational factors in order to gain further knowledge on successful rehabilitation and return to work.

\section{Conclusions}

We found that referral rates to MMR were positively associated with PHCCs located in medium and large communities with higher socioeconomic status among the registered population, private PHCCs and PHCCs providing their own MMR. Patients with MSD are thus facing significant inequities and were thus not offered the same opportunities for referrals to rehabilitation regardless of which $\mathrm{PHCC}$ they visited.

\section{Abbreviations}

ACG: Adjusted Clinical Groups; CNI: Care Need Index; MMR: Multimodal rehabilitation; MSD: Musculoskeletal disorders; PHC: Primary health care; PHCC: Primary health care centre

\footnotetext{
Acknowledgements

Senior lecturer, PhD, Anna Lindgren, Centre for Mathematical Sciences, Lund University, Lund, Sweden, supported data analysing and interpretation. Administrative personnel at Department of Healthcare Governance, Malmö, supported retrieving PHCCs data. Dorthe Geisler helped editing tables and figures. Dr Gwenllian Wynne-Jones, Primary Care \& Health Sciences, Keele University UK, supported with revision concerning concepts and English language of the manuscript. Adam Post performed data validation and proofreading of the manuscript.
} 


\section{Funding}

This study was funded within the PhD studies of CPS, Lund University, Lund, Sweden.

\section{Availability of data and materials}

The datasets generated during and/or analysed the current study are not publicly available due to this data material belongs to the Regional Council Skåne, but are available from the corresponding author on reasonable request.

\section{Authors' contributions}

CPS, BG and SH were responsible for the study design, data analysis and interpretation. CPS collected and prepared data and was major contributor in writing the manuscript. KS, IP, MF and AN took part in study design and interpretation of data. All authors read and approved the final manuscript.

\section{Competing interests}

The authors declare that they have no competing interests.

\section{Consent for publication}

Not applicable.

\section{Ethics approval and consent to participate}

This study has been approved by the Regional Ethical Review Board in Lund, Dnr 2014/290. In Region Skåne the PHCCs are obligated to participate in follow-ups on quality of care.We had agreements from the Regional Council Skåne to evaluate and study health care provider factors associated to referring to MMR

\section{Author details}

${ }^{1}$ Faculty of Medicine, Department of Clinical Sciences Lund, Orthopedics, Lund University, Lund, Sweden. 'Epidemiology and Register Centre South, Region Skåne, Lund, Sweden. ${ }^{3}$ Department of Research and Development, Region Kronoberg, Växjö, Sweden. ${ }^{4}$ Division of Occupational and Environmental Medicine, Institute of Laboratory Medicine, Lund University, Lund, Sweden. ${ }^{5}$ Department of Health Sciences, Physiotherapy, Lund University, Lund, Sweden. ${ }^{6}$ Blekinge Centre of Competence, Karlskrona, Sweden. ${ }^{7}$ Skåne Regional Council, Region Skåne, Department of Healthcare Governance, Malmö, Sweden.

\section{Received: 15 October 2015 Accepted: 14 December 2016} Published online: 07 January 2017

\section{References}

1. Alexanderson K, Norlund A. Swedish Council on Technology Assessment in Health Care (SBU). Chapter 12. Future need for research. Scand J Public Health Suppl. 2004;63:256-8.

2. Jordan KP, Kadam UT, Hayward R, Porcheret M, Young C, Croft P. Annual consultation prevalence of regional musculoskeletal problems in primary care: an observational study. BMC Musculoskelet Disord. 2010;11:144.

3. Hasselstrom J, Liu-Palmgren J, Rasjo-Wraak G. Prevalence of pain in general practice. Eur J Pain. 2002;6(5):375-85.

4. Vos T, Flaxman AD, Naghavi M, Lozano R, Michaud C, Ezzati M, Shibuya K Salomon JA, Abdalla S, Aboyans V, et al. Years lived with disability (YLDs) for 1160 sequelae of 289 diseases and injuries 1990-2010: a systematic analysis for the Global Burden of Disease Study 2010. Lancet. 2012;380(9859):2163-96.

5. Murray CJL, Vos T, Lozano R, Naghavi M, Flaxman AD, Michaud C, Ezzati M, Shibuya K, Salomon JA, Abdalla S, et al. Articles: Disability-adjusted life years (DALYs) for 291 diseases and injuries in 21 regions, 1990-2010: a systematic analysis for the Global Burden of Disease Study 2010. Lancet. 2012;380:2197-223.

6. Thulesius HO, Grahn BE. Reincentivizing-a new theory of work and work absence. BMC Health Serv Res. 2007;7:100.

7. The National Board of Health and Welfare S. Nationella riktlinjer rörelseorganens sjukdomar, (National guidelines musculoskeletal diseases). 2012. In Swedish. In. https://www.socialstyrelsen.se/Lists/Artikelkatalog/ Attachments/18665/2012-5-1.pdf. Accessed 28 Sept 2015.

8. Joud A, Petersson IF, Englund M. Low back pain: epidemiology of consultations. Arthritis Care Res (Hoboken). 2012;64(7):1084-8.

9. The National Board of Health and Welfare S. God vård, (National indicators of good care). In Swedish. In. https://www.socialstyrelsen.se/publikationer2009/ nationellaindikatorerforgodvard. Accessed 14 Feb 2016.
10. Busch $H$, Bodin L, Bergström $G$, Jensen IB. Patterns of sickness absence a decade after pain-related multidisciplinary rehabilitation. Pain. 2011;152(8):1727-33.

11. Norlund A, Ropponen A, Alexanderson K. Multidisciplinary interventions: review of studies of return to work after rehabilitation for low back pain Rehabil Med. 2009;41(3):115-21.

12. Lin CW, Haas M, Maher CG, Machado LA, van Tulder MW. Cost-effectiveness of guideline-endorsed treatments for low back pain: a systematic review. Eur Spine J. 2011;20(7):1024-38.

13. Jensen IB, Busch H, Bodin L, Hagberg J, Nygren A, Bergstrom G. Cost effectiveness of two rehabilitation programmes for neck and back pain patients: A seven year follow-up. Pain. 2009;142(3):202-8.

14. Kuoppala J, Lamminpaa A, Husman P. Work health promotion, job well-being, and sickness absences-a systematic review and meta-analysis. J Occup Environ Med. 2008;50(11):1216-27.

15. Williams RM, Westmorland MG, Lin CA, Schmuck G, Creen M. Effectiveness of workplace rehabilitation interventions in the treatment of work-related low back pain: A systematic review. Disabil Rehabil. 2007;29(8):607-24.

16. Regions SAoLAa. Rehabiliteringsgarantin 2008 (The national rehabilitation program). In Swedish. In. http://skl.se/tjanster/omwebbplatsen/sok.23. html?q=rehabiliteringsgarantin\&submitButton.x=0\&submitButton.y=0. Accessed 20 Apr 2015

17. Regions SAoLAa. Rehabiliteringsgarantin 2012 (The national rehabilitation program). In Swedish. In. http://skl.se/tjanster/omwebbplatsen/sok.23. html?q=rehabiliteringsgarantin\&submitButton. $x=0 \&$ submitButton. $y=0$. Accessed 5 Apr 2015.

18. Bramberg EB, Klinga C, Jensen I, Busch H, Bergstrom G, Brommels M, Hansson J. Implementation of evidence-based rehabilitation for non-specific back pain and common mental health problems: a process evaluation of a nationwide initiative. BMC Health Serv Res. 2015:15:79.

19. Busch H, Bonnevier H., Hagberg J., Lohela Karlsson M., Bodin L., Norlund A., Jensen I.: En nationell utvärdering av rehabiliteringsgarantins effekter på sjukfrånvaro och hälsa, Slutrapport del I. (A national evaluation of the rehabilitation guarantee effects on sick leave and health). In Swedish. In.: Enheten för interventions- och implementeringsforskning, Institutet för miljömedicin (IMM), Karolinska Institutet, Stockholm; 2011.

20. Bakshi A HJL, Brommels M, Klinga C, Bonnevier H, Jensen I B: En processutvärdering av implementeringen av den nationella rehabiliteringsgarantin. Slutrapport del II. (A process evaluation of the implementation of the national rehabilitation guarantee). In Swedish. In.: Enheten för implementerings- och interventionsforskning, Institutet för miljömedicin samt Medical management Centrum, Institutionen för lärande information och etik, Karolinska Institutet. Stockholm; 2011

21. SBU Safhtaaafss. Acute neck and back pain: preventive interventions. Effects of physical training, manual treatment and cognitive behavioral interventions, 2016. In., vol. 2016. http://www.sbu.se/en/publications/sbuassesses/acute-neck-and-back-pain-preventive-interventions-effects-ofphysical-training-manual-treatment-and-cognitive-behavioral-interventions/.

22. Stigmar KG, Petersson IF, Joud A, Grahn BE. Promoting work ability in a structured national rehabilitation program in patients with musculoskeletal disorders: outcomes and predictors in a prospective cohort study. BMC Musculoskelet Disord. 2013;14(1):57.

23. Holmberg S, Ekström H. Nöjdare patienter ... personalen tycker att det blivit sämre, (Primary health care in Kronoberg before and after the care choice. More satisfied patients ... the staff think it got worse) . In Swedish. Lakartidningen. 2014;111(CSUU 2014):25-6.

24. Hedin K, Andre M, Hakansson A, Molstad S, Rodhe N, Petersson C. A population-based study of different antibiotic prescribing in different areas. Br J Gen Pract. 2006;56(530):680-5.

25. Anell A, Glenngard AH, Merkur S. Sweden health system review. Health Syst Transit. 2012;14(5):1-159.

26. Isaksson D, Blomqvist $P$, Winblad U. Free establishment of primary health care providers: effects on geographical equity. BMC Health Serv Res. 2016;16:28.

27. Neutens T. Accessibility, equity and health care: review and research directions for transport geographers. J Transport Geography. 2015;43:14-27.

28. Dewulf B, Neutens T, De Weerdt Y, Van de Weghe N. Accessibility to primary health care in Belgium: an evaluation of policies awarding financial assistance in shortage areas. BMC Fam Pract. 2013:14:122.

29. Campbell JL, Ramsay J, Green J. Practice size: impact on consultation length, workload, and patient assessment of care. Br J Gen Pract. 2001:51(469):644-50. 
30. Ekström H, Holmberg S, Karlsson A: Vårdval Kronoberg (Primary care choise reform in Kronoberg, Sweden) FoU-rapport 2013:2. In Swedish. In. http://www.fouvalfard.se/file/2013-vardval-kronoberg.pdf.

31. Andersson F, Janlöv, N., Rehnberg, C.: Konkurrens, kontrakt och kvalitet hälso- och sjukvård i privat regi (Competition, contracts and quality healthcare privately). In Swedish. 2014:5. In. http://www.vardanalys.se/ Global/Rapporter\%20pdf-filer/2014/2014-5\%20Konkurrens, \%20kontrakt\%20och\%20kvalitet\%20-\%20h\%C3\%A4lso-\%20och\% 20sjukv\%C3\%A5rd\%20i\%20privat\%20regi.pdf. Accessed 5 Sept 2016.

32. Gulliford MC, Mahabir D. Utilisation of private care by public primary care clinic attenders with diabetes: relationship to health status and social factors. Soc Sci Med. 2001;53(8):1045-56.

33. Analysis VTSAfHaCS: Vårdval och jämlik vård inom primärvården(Vårdval and equal treatment in primary care). In Swedish. In. http://www.vardanalys.se/ Rapporter/2015/Vardval-och-jamlik-vard-inom-primarvarden-En-jamforandestudie-mellan-tre-landsting-fore-och-efter-vardvalets-inforande/. Accessed 5 Sept 2016.

34. Starfield B, Weiner J, Mumford L, Steinwachs D. Ambulatory care groups: a categorization of diagnoses for research and management. Health Serv Res. 1991;26(1):53-74.

35. Weiner JP, Starfield BH, Steinwachs DM, Mumford LM. Development and application of a population-oriented measure of ambulatory care case-mix. Med Care. 1991;29(5):452-72.

36. Carlsson L, Borjesson U, Edgren L. Patient based 'burden-of-illness' in Swedish primary health care. Applying the Johns Hopkins ACG case-mix system in a retrospective study of electronic patient records. Int J Health Plann Manage. 2002;17(3):269-82.

37. Malmstrom M, Sundquist J, Bajekal M, Johansson SE. Indices of need and social deprivation for primary health care. Scand J Soc Med. 1998;26(2):124-30

38. Sundquist K, Malmström M, Johansson S-E, Sundquisr J. Care Need Index, a useful tool for the distribution of primary health care resources. J Epidemiol Community Health. 2003;57:347-52.

39. Sennehed C. NA, Holmberg S., Stigmar K., Petersson I., Forsbrand M., Hallgårde U., Grahn B. Multimodal smärtrehabilitering, vårdgivarrelaterade faktorers betydelse för remittering (Multimodal pain rehabilitation, caregiver related factors for referrals). In Swedish. In.: Lunds Universitet, Region Skåne, Sweden, 2015

40. (Analysis) MfvTSAfHaCS: Vem vill veta vad för att välja (Who wants to know what to choose). 2014. In Swedish. In., vol. 2014:1. http://www.vardanalys.se/ Rapporter/2014/Nem-vill-veta-vad-for-att-valja/.

41. Analysis TSAfHaCS: En mer jämlik vård är möjlig. Analys av omotiverade skillnader i vård, behandling och bemötande (A more equitable care is possible. Analysis of unjustified disparities in care, treatment and attitudes). 2014. In Swedish. In., vol. 2014:7. http://www.vardanalys.se/Global/ Rapporter\%20pdf-filer/2014/2014-7-En\%20mer\%20j\%c3\%a4mlik\%20v\% c3\%a5rd\%20\%c3\%a4r\%20m\%c3\%b6jlig_webb.pdf. Accessed 5 Oct 2015

42. Malmstrom M, Sundquist J, Johansson SE. Neighborhood environment and self-reported health status: a multilevel analysis. Am J Public Health. 1999;89(8):1181-6.

43. The National Board of Health and Welfare S: Om primärvårdens domän och strategier (The primary health care, domain and strategies). 2004. In Swedish. In. http://www.socialstyrelsen.se/publikationer2004/2004-123-19. Accessed 24 Apr 2015

44. Hellman TBH, Jensen I, Hagberg J, Busch H, Björk Brämberg E, Bergström G. En processutvärdering av implementeringen av den nationella rehabiliteringsgarantin. Slutrapport, (A process evaluation of the implementation of the national rehabilitation guarantee, Final report part II). In Swedish. In.: Enheten för interventions- och implementeringsforskning, Institutet för miljömedicin (IMM). Karolinska Institutet; 2014.

45. Hellman T, Jensen I, Bergström G, Busch H. Returning to work - a long-term process reaching beyond the time frames of multimodal non-specific back pain rehabilitation. Disabil Rehabil. 2015;37(6):499-505.

46. Rothman MGMD, Ortendahl MMDP, Rosenblad AP, Johansson A-CPTP. Improved quality of life, working ability, and patient satisfaction after a pretreatment multimodal assessment method in patients with mixed chronic muscular pain: a randomized-controlled study. Clin J Pain. 2013;29(3):195-204.

47. Farr M, Cressey P. Understanding staff perspectives of quality in practice in healthcare. BMC Health Serv Res. 2015;15:123.
48. Pflueger D. Accounting for quality: on the relationship between accounting and quality improvement in healthcare. BMC Health Serv Res. 2015;15(1):178.

49. Phillips CB, Dwan K, Hepworth J, Pearce C, Hall S. Using qualitative mixed methods to study small health care organizations while maximising trustworthiness and authenticity. BMC Health Serv Res. 2014;14(1):559.

50. Utredningar SO: Effektiv vård (Efficient health care) 2016. In Swedish. In. http://www.regeringen.se/contentassets/42b0aef4431c4ebf9410b8ee771830eb/ effektiv-vard-slutbetankande-av-en-nationell-samordnare-for-effektivareresursutnyttjande-inom-halso-och-sjukvarden_sou-2016-2.pdf. Accessed 5 Sept 2016.

51. Gatchel RJ, Peng YB, Peters ML, Fuchs PN, Turk DC. The biopsychosocial approach to chronic pain: scientific advances and future directions. Psychol Bull. 2007;133(4):581-624.

52. Olaya-Contreras $P$, Styf J. Biopsychosocial function analyses changes the assessment of the ability to work in patients on long-term sick-leave due to chronic musculoskeletal pain: the role of undiagnosed mental health comorbidity. Scand J Public Health. 2013;41(3):247-55.

53. Gustafsson K, Aronsson G, Marklund S, Wikman A, Hagman M, Floderus B. Social integration, socioeconomic conditions and type of ill health preceding disability pension in young women: a Swedish population-based study. Int J Behav Med. 2013.

54. Ropponen A, Svedberg P, Koskenvuo M, Silventoinen K, Kaprio J. Physical work load and psychological stress of daily activities as predictors of disability pension due to musculoskeletal disorders. Scand J Public Health. 2014;42(4):370-6.

55. Karkkainen S, Pitkaniemi J, Silventoinen K, Svedberg P, Huunan-Seppala A, Koskenvuo K, Koskenvuo M, Alexanderson K, Kaprio J, Ropponen A. Disability pension due to musculoskeletal diagnoses: importance of work-related factors in a prospective cohort study of Finnish twins. Scand J Work Environ Health. 2013;39(4):343-50.

56. Ropponen A, Silventoinen K, Svedberg P, Alexanderson K, Huunan-Seppälä A, Koskenvuo K, Koskenvuo M, Kaprio J. Effects of work and lifestyle on risk for future disability pension due to low back diagnoses: a 30-year prospective study of Finnish twins. J Occup Environ Med. 2012;54(11):1330-6.

57. Ropponen A, Svedberg P, Huunan-Seppälä A, Koskenvuo K, Koskenvuo M, Alexanderson K, Silventoinen K, Kaprio J. Personality traits and life dissatisfaction as risk factors for disability pension due to low back diagnoses: a 30-year longitudinal cohort study of Finnish twins. J Psychosom Res. 2012;73(4):289-94.

\section{Submit your next manuscript to BioMed Central and we will help you at every step:}

- We accept pre-submission inquiries

- Our selector tool helps you to find the most relevant journal

- We provide round the clock customer support

- Convenient online submission

- Thorough peer review

- Inclusion in PubMed and all major indexing services

- Maximum visibility for your research

Submit your manuscript at www.biomedcentral.com/submit
Biomed Central 